Ener gy- and- pi t ch- angl e- resol ved escapi ng beam $i$ on measur ement $s$ by Far aday- cup-based fast- $i$ on I oss det ect or i $n$ Wendel st ei $n$ 7- $X$

\begin{tabular}{|l|l|}
\hline $\begin{array}{l}\text { j our nal or } \\
\text { publ i cat i on t i l e }\end{array}$ & Jour nal of I nst r ument ai on \\
\hline vol une & 14 \\
\hline page range & Co9021 \\
\hline year & $2019-09-13$ \\
\hline URL & ht t p: //hdl . handl e. net /10655/00012561 \\
\hline
\end{tabular}




\title{
Energy-and-Pitch-Angle-Resolved Escaping Beam Ion Measurements by Faraday-cup-based Fast-Ion Loss Detector in Wendelstein 7-X
}

\author{
K. Ogawa, ${ }^{\mathrm{a}, b,{ }^{*}}$ S. A. Bozhenkov ${ }^{\mathrm{c}}$ S. Äkäslompolo, ${ }^{\mathrm{c}}$ C. Killer, ${ }^{\mathrm{c}}$ O. Grulke, ${ }^{\mathrm{c}}$ \\ D. Nicolai, ${ }^{d}$ G. Satheeswaran, ${ }^{d}$ M. Isobe, ${ }^{\mathrm{a}, \mathrm{b}}$ M. Osakabe, ${ }^{\mathrm{a}, \mathrm{b}}$ M. Yokoyama, ${ }^{\mathrm{a}, \mathrm{b}}$ \\ R. C. Wolf, ${ }^{\mathrm{C}}$ and the W7-X team $^{\mathrm{c}}$ \\ ${ }^{a}$ National Institute for Fusion Science, National Institutes of Natural Sciences, \\ Toki, Japan \\ ${ }^{b}$ SOKENDAI (The Graduate University for Advanced Studies), \\ Toki, Japan \\ ${ }^{c}$ Max-Planck-Institute for Plasma Physics, \\ Greifswald, Germany \\ ${ }^{d}$ Institute of Energy and Climate Research Plasma Physics IEK-4, \\ Forschungszentrum Jülich, Germany \\ E-mail: kogawa@nifs.ac.jp
}

ABSTRACT: With the objective of understanding the energetic-particle loss mechanism in threedimensional plasmas, a Faraday-cup-based fast-ion loss detector (FILD) was developed and installed in OP1.2b in Wendelstein 7-X (W7-X) as a collaboration between the National Institute for Fusion Science and Max-Planck-Institute for Plasma Physics. The FILD, which consists of double apertures and thin aluminum foils, was based on a magnetic spectrometer using the magnetic field of the fusion device. The double aperture limits the thermal ion, but allows the energetic ion to enter the FILD box. The thin aluminum foils serve as the ion collector. Orbitfollowing calculations were performed in order to find a suitable position for the FILD. The results indicated that barely co- and counter-going transit ions reached the FILD position mounted on the multi-purpose manipulator installed on W7-X. Moreover, because the injection angle of neutral beams injector installed on W7-X was relatively perpendicular, the target range of pitch angle was set to from 91 degrees to 150 degrees. An energy-and-pitch-angle map was created using a grid calculation code in order to decide on the position/size of aperture and aluminum foil. The grid calculation code indicates the position where the energetic ion would strike on the aluminum foil. Here, the map was used as the basis for designing an aluminum foil pattern with two energies and four pitch angle ranges. Also, note that the lower energy range was designed so that it accommodates a neutral beam injection energy of $55 \mathrm{kV}$. Measurements of beam ion losses were performed in neutral beam (NB) blip experiments, where concurrent increases and decreases of barely co-going transit beam ion losses due to NB injections were observed. Furthermore, this study validated that the FILD installed on a midplane manipulator probe is capable of probing a range of radii spanning $1.0-1.5 \mathrm{~cm}$, over which the beam ion loss current would vary significantly.

KEYWORDS: Beam ion confinement; Fast-ion loss detector; Wendelstein 7-X; Faraday-cup; NB blip. 


\section{Contents}

1. Introduction 1

2. Feasibility study of FILD installation 1

3. Faraday-cup-based fast-ion loss detector 3

4. Initial results of beam ion loss measurements in W7-X 4

5. Summary 6

\section{Introduction}

The study of fast-ion losses due to magnetic field ripples and magnetohydrodynamic (MHD) modes is an important research topic regarding fusion-born energetic-alpha losses in a fusion reactor [1]. Faraday-cup-based fast-ion loss detectors (FILDs) have been previously developed and installed for measurements of beam ion loss flux in stellarators, such as Compact Helical System (CHS) [2, 3] and Heliotron-J [4, 5], as well as tokamaks such as the National Spherical Torus eXperiment (NSTX) [6], the Joint European Torus (JET) [7, 8], and the Doublet III-D (DIII-D) [9]. The Faraday-cup-based FILD, which is relatively cost-effective in construction compared to the scintillator-based FILD [10], is capable of pitch-angle- $(\chi)$-and-energy- $(E)$ resolved measurement of the absolute flux of fast ions, thus giving a clear understanding of fastion loss processes due to MHD modes and/or non-axisymmetric magnetic field ripples. For example, an increase of co-going beam ion losses due to energetic-particle modes and toroidal Alfvén eigenmodes was reported in CHS [3]. In order to understand fast-ion losses in Wendelstein 7-X (W7-X) plasmas, a numerical study of fast-ion loss was performed [11] and an installation of fast-ion loss diagnostics was planned and reported in 2002 [12]. In the OP1.2b campaign of W7-X experiments, neutral beam (NB) injection became available, enabling the experiment team to study the fast-ion confinement in W7-X. A Faraday-cup-based FILD has been designed and installed as a joint cooperative project between the National Institute for Fusion Science (NIFS) and Max-Planck-Institute for Plasma Physics. More specifically, this manuscript reports the design study of FILD for W7-X and the initial results of beam ion loss measurements.

\section{Feasibility study of FILD installation}

W7-X is one of the world's largest fusion devices of the stellarator type with a major radius of $5.5 \mathrm{~m}$ and an averaged minor radius of $0.5 \mathrm{~m}$ (Fig. 1(a)). W7-X is equipped with electron cyclotron resonance heating $(\mathrm{ECRH})$ and NB injections as heating devices [13]. Here the acceleration voltage and injection power of $\mathrm{NB}$ are $55 \mathrm{keV}\left(\right.$ for $\left.\mathrm{H}^{+}\right)$and up to $1.7 \mathrm{MW}$, respectively [14]. In the OP1.2b campaign, NB injection with two ion sources (NB\#7 and NB\#8) became available from August 2018. A multi-purpose manipulator (MPM) was installed with the aim of studying the behavior of a plasma edge region [15]. As the name suggests, MPM's head part can be 
changed between experimental days due to the specified interface. NIFS Lorentz orbit code (LORBIT) [16] was applied to find which MPM location is suitable for the beam ion loss
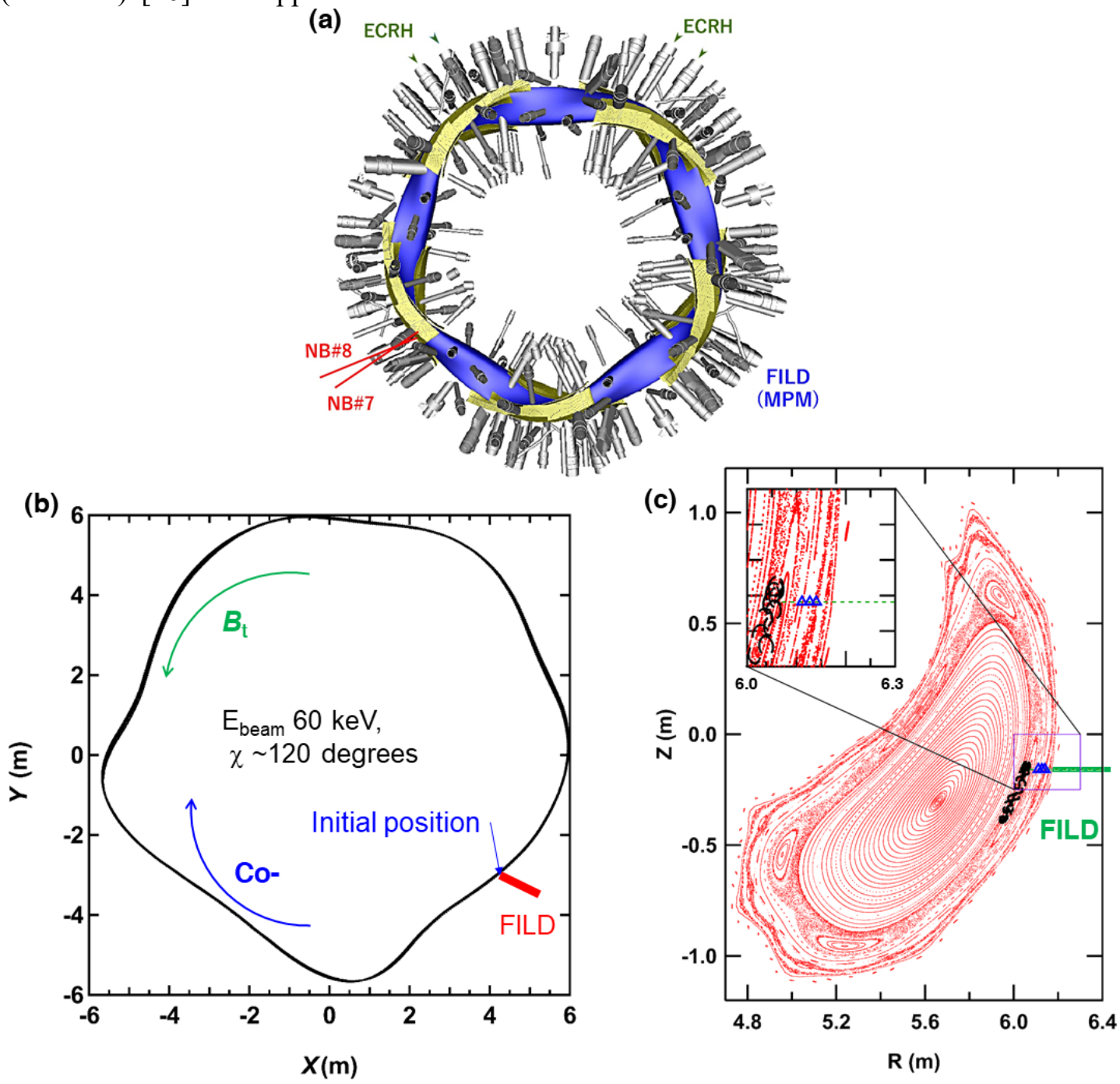

Figure 1. (a) Bird's-eye view of W7-X with ECRH, NB\#7, NB\#8, and FILD port. Top view (b) and Poincaré plot (c) of co-going transit beam ion orbit reaching the FILD calculated by the LORBIT code.

measurement. By principle, the LORBIT code is based on the equation of motion of a charged particle in the Cartesian coordinates, without considering collisions. The FILD positions, together with a typical co-going beam ion orbit as seen from the top and the Poincare plot of the beam ion, are depicted in Figs. 1(b) and 1(c). Here, the start position of the orbit was $(x, y, z)=(-5.64 \mathrm{~m}$, $-2.24 \mathrm{~m},-0.157 \mathrm{~m})$. The values of $E$ and $\chi$ of the particle at the FILD position was $55 \mathrm{keV}$ and 120 degrees, respectively. Note that the calculation was performed in the standard W7-X magnetic configuration assuming a counterclockwise direction of the toroidal magnetic field as seen from the top. The orbit of beam ions injected by NB\#8 was followed up to $1 \mathrm{~ms}$ in order to determine whether the beam ions would reach the FILD position. Accordingly, the number of calculated beam ions was $5 \times 10^{4}$, and the FILD head location was set along the path of the MPM insertion. Note that the diameter of the FILD head was set at $100 \mathrm{~mm}$. Figures 2(a) and 2(b) show the 
number of particles reaching the FILD as a function of the radial FILD position $R$ and the distribution of $\chi$ at the radial position of FILD $R$ of 5.95-6.00, 6.00-6.05, and 6.05-6.10 m. In particular, the number of particles continuously decreased with greater $R$, and only a small number of beam ions were able to reach the detector at $R>6.1 \mathrm{~m}$. These calculation results indicate that beam ions having $\chi$ from 50 degrees to 70 degrees (barely counter-going transit ion) and $\chi$ from 100 degrees to 110 degrees (barely co-going transit ion) reached the FILD position. For pitch angle, the distribution was slightly different in $R$ of 5.95-6.00 m, relative to the other cases. Moreover, heat flux distribution due to the beam ions evaluated by ASCOT codes, as a guiding center orbit-following code with collisions [17], showed that heat flux of up to $1 \mathrm{MW} / \mathrm{m}^{2}$ reached the FILD head (Fig. 2(c)).

\section{Faraday-cup-based FILD}

The FILD head designed for W7-X appears in Fig. 3(a), with outer radius and head length of approximately 80 and $92 \mathrm{~mm}$, respectively. The FILD was designed to enable the measurement of helically trapped and co-going beam ions. The detector head was made of molybdenum in order to resist high-heat load from the plasma. The head has an aperture consisting of front and rear apertures that restrict the orbit of energetic ions entering the probe. Moreover, the FILD head was equipped with a MPM adapter for installation at the MPM arm. The graphite cover surrounding the head was added to prevent heat damage on the molybdenum head and to monitor the probe surface temperature with an infrared camera. Note that part of the graphite head was cut away in order to reduce the self-shadow effect. The Faraday films located inside the molybdenum head functioned as charge collectors. A Faraday film is a thin film, normally having thickness of $0.2 \mu \mathrm{m}$ of aluminum vapor deposited onto one side of the quartz substrate. The

Figure 2. (a) Number of particles reaching the FILD position as evaluated by the LORBIT code. (b) Pitch angle distribution of the particles that reached the FILD position. (c) Heat flux distribution on the FILD head, as evaluated by the ASCOT code.

Figure 3. (a) Schematic drawing of the FILD head. (b) Energy-and-pitch-angle map on the FILD head. The FILD is designed to measure co-going beam ions. (c) Electrical diagram of FILD.

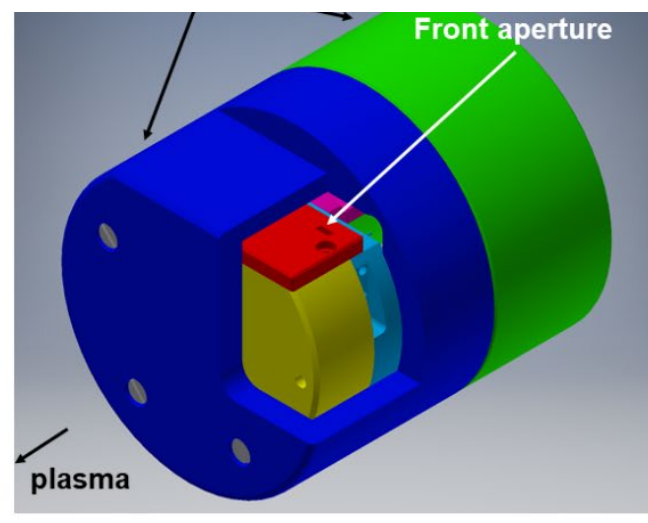

(c)

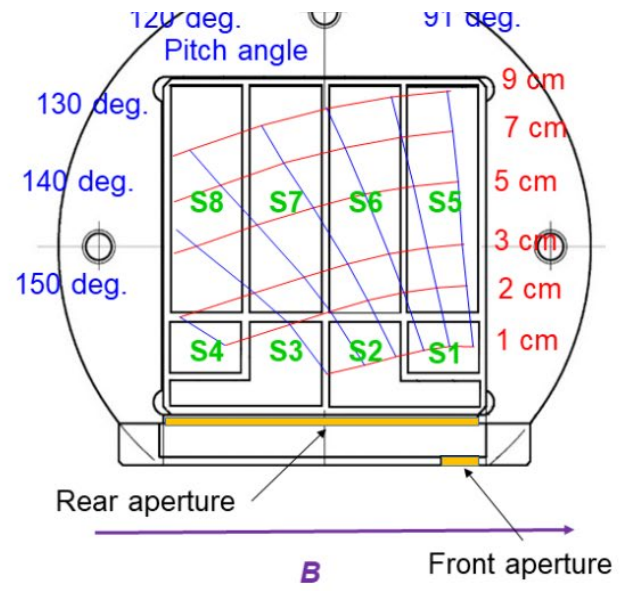

B
Isolation amplifier HIS-LV, DEWETRON

Gain: 1

Cutoff freq.: $1 \mathrm{kHz}$
Data acquisition system $A C Q 132 \mathrm{CPCl}$

D-TACQ Solutions Ltd.

Sampling freq.: $500 \mathrm{kHz}$ 
geometry of the apertures and the Faraday films were decided by the energy vs. pitch grid calculation program developed in Princeton Plasma Physics Laboratory (PPPL) [10]. The width and the height of the front aperture were 4.0 and $1.6 \mathrm{~mm}$, respectively. Those of the rear aperture were accordingly 39.0 and $0.8 \mathrm{~mm}$. Both apertures had a 4-mm gap and were located $1 \mathrm{~mm}$ above the Faraday films. The layout of Faraday films was also decided with reference to the result of the grid calculation program. Furthermore, two energies and four pitch angle ranges were designed, as depicted in Fig. 3(b). Here, the energy Larmor radius of 1 and $2 \mathrm{~cm}$ corresponded to the beam energy of 21 and $84 \mathrm{keV}$, respectively. The energy Larmor radius and pitch angle range of each film are listed in Table 1 . As the injection energy was $55 \mathrm{keV}$, it was expected that some signals might appear on channels S1 to S4 in the NB-heated W7-X plasma. Contact pins (F112,

Table 1 Detectable energy Larmor radius and pitch angle range for each foil.

\begin{tabular}{|l|c|c|c|c|c|c|c|c|}
\hline Foil name & S1 & S2 & S3 & S4 & S5 & S6 & S7 & S8 \\
\hline Larmor radius (cm) & $\sim 1$ & 1 & 1 & 2 & 2 & 2 & 2 & 3 \\
& & - & - & - & - & - & - & - \\
& & 2 & 2 & 3 & 9 & 9 & 9 & 9 \\
\hline Pitch angle (deg) & 91 & 120 & 140 & $\sim 150$ & 91 & 110 & 130 & $\sim 150$ \\
& - & - & - & & - & - & - & \\
& 120 & 140 & 150 & & 110 & 130 & 140 & \\
\hline
\end{tabular}

TESPRO Co. Ltd.) were used to connect the Faraday films and the conduction wires. Figure 3(c) illustrates the block diagram of the FILD system. Here, electrical current from each Faraday film is carried to the low-input impedance current amplifier (I-76, NF Corporation, a gain of $10^{4} \mathrm{~V} / \mathrm{A}$ ). The current amplifier is driven by a 9-V battery (6LF22) to reduce the $50 \mathrm{~Hz}$ AC noise. Note that the amplitude of alternating current noise having frequency of $50 \mathrm{~Hz}$ in each channel was largely suppressed after the $9-\mathrm{V}$ battery was applied for the current amplifier. Additionally, the amplitude of alternating current noise exceeded the input range of data acquisition system $( \pm 6 \mathrm{~V})$ after power was supplied from the $220-\mathrm{V}$ alternating current. Moreover, the amplitude was reduced to at most $\pm 2 \mathrm{~V}$ after the $9-\mathrm{V}$ battery was used. The authors found that the main components of noise were contaminated between the Faraday films and the input cable of the current amplifier. To date, they had no methods for determining how the noise could be avoided. Subsequently, the voltage signal was fed into an isolation amplifier (HIS-LV, DEWETRON), whose gain and the cutoff (low-pass) frequency were both set at $1 \mathrm{kHz}$. The relatively low cutoff frequency was chosen in order to suppress the relatively high-frequency electrical noise. The signal was acquired via the data acquisition system (ACQ132CPCI, D-TACQ Solutions Ltd.) at a sampling frequency of 500 $\mathrm{kHz}$, which is the typical speed.

\section{Initial results of beam ion loss measurements in W7-X}

In the NB blip experiments, beam ion losses were measured in standard configurations in W7-X (Fig. 4(a)). In the discharge, short-pulse NBs 10-ms wide and separated by 100-ms interval were injected into the ECRH-heated plasma. Throughout the discharge, the central electron temperature $\left(T_{\mathrm{e} 0}\right)$ measured by Thomson scattering was approximately $4 \mathrm{keV}$, while the lineaveraged density ( $n_{\text {e__avg }}$ ) measured by interferometer was about $5 \times 10^{19} \mathrm{~m}^{-3}$. Moreover, during the discharge the position of the FILD head was varied in three steps as follows: $R=6.140,6.127$, and $6.111 \mathrm{~m}$, with $R=0$ as the torus center. Gray lines at bottom right in Fig. 4(a) indicate the raw signals of FILD, whereas the red and blue lines each indicate a filtered signal. For filtered signal, a numerical band-stop filter and a smoothing filter were applied for removal of the 
electromagnetic noise that was contaminated with the real signal. The frequency range of bandstop filter was 48 to $52 \mathrm{~Hz}$, which was deemed sufficient to remove the $50-\mathrm{Hz}$ alternating current
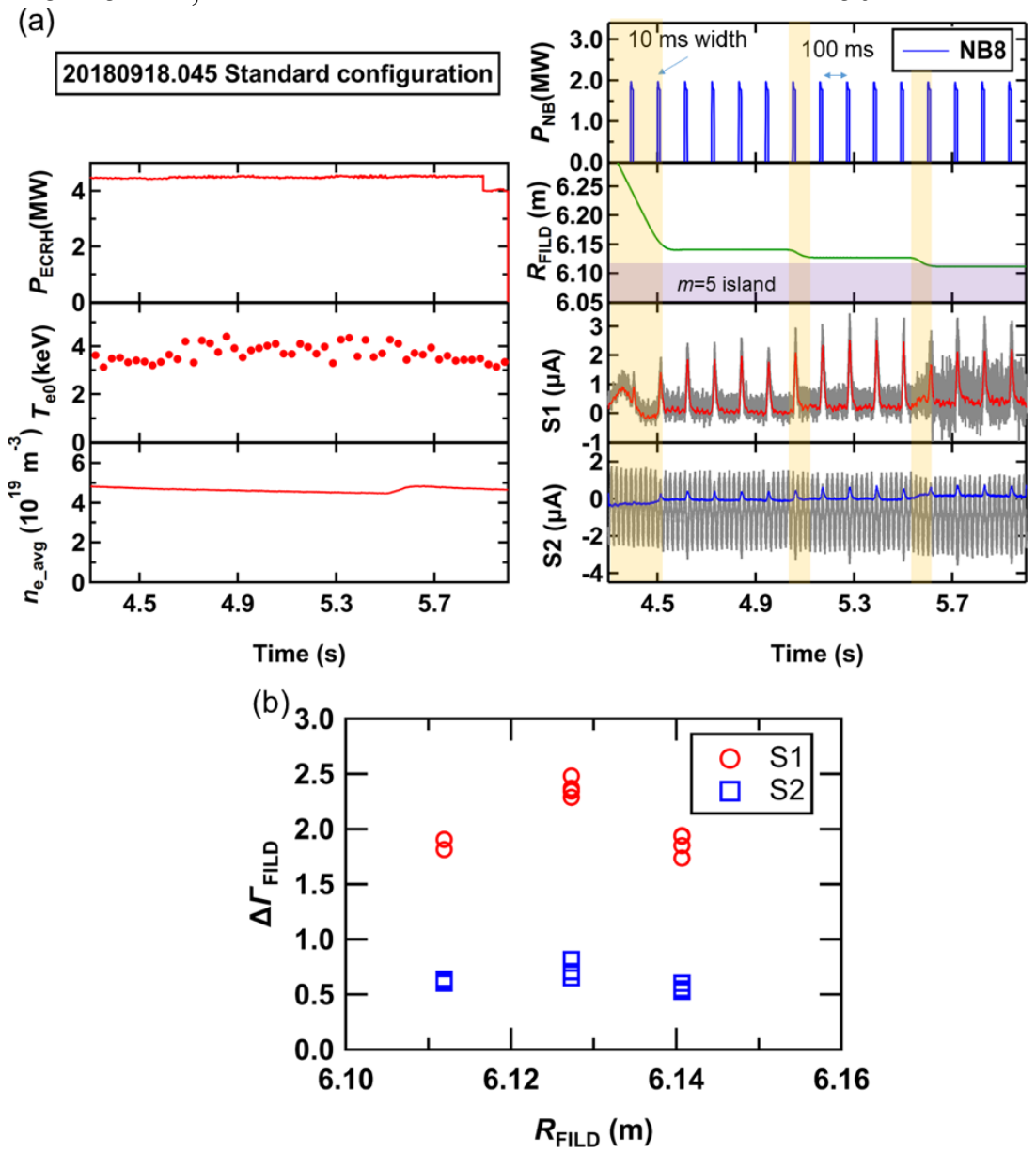

Figure 4. (a) Time evolution of the short-pulse NB injection experiment. (b) Increment of beam-ion loss signal as a function of the FILD position.

noise, whereas there were 2000 points (4-ms time resolution) of smoothing filter. In the lower energy channel, the beam ion loss current corresponding to $\chi$ of 91-140 was successfully measured. The energy-and-pitch-angle range measured by FILD was consistent with the expected range by LORBIT calculation and beam injection energy. In addition, it is worth noting that no significant signal was observed in channels S3 to S8. On the one hand, no signal was expected in S5 to S8 as those films were used for detecting. On the other hand, the absence of significant signal in S3 and S4 range of S3 and S4 (140 degrees to 150 degrees), reached the FILD position. No signal in S3 and S4 was consistent with that predicted by the LORBIT code, as previously shown in Fig. 2(b), where beam ions having a pitch angle of 100 degrees to 110 degrees, which is the out-of-the-pitch-angle range of S3 and S4. The typical signal level of S1 and S2 were 2-3 and $0.5-1 \mu \mathrm{A}$, respectively, which were comparable to the signal level of CHS and Heliotron-J $[3,5]$. Apparently, the measurement of absolute current of the energetic ion is an advantage exhibited by this Faraday-cup-type FILD, as compared to the scintillator type. The FILD signals S1 and S2 varied with the head position of FILD. Here, background fluctuations of the signal became discernible especially at $R=6.11 \mathrm{~m}$. Such relatively strong background fluctuations could be attributed to the effect of plasma potential, as depicted in Fig. 1(c), in which this head position 
of FILD was almost inside the edge magnetic island of $m=5$ structure. Therefore, the plasma potential may have affected the electrical potential of the signal or the ground line of FILD. Concerning the relatively high-noise level, a discussion of energetic ion loss caused by static or quasi-static is possible. On the contrary, a discussion of energetic-ion-driven or bulk-plasmapressure-driven MHD-mode-induced loss will almost be impossible using this current system. To enhance the understanding of MHD-driven energetic ion loss, there is a need to establish a solution for the noise issues or to install a scintillator-type FILD. Figure 4(b) shows the increment of the FILD signal due to the NB injection $\left(\Delta \Gamma_{\mathrm{FILD}}\right)$, as a function of the FILD position in discharge $\# 20180918.045$. Here, the radial profile of $\Delta \Gamma_{\text {FILD }}$ demonstrated that $\Delta \Gamma_{\text {FILD }}$ had a peak at around $R_{\text {FILD }}$ of $6.127 \mathrm{~m}$ in $\mathrm{S} 1$ and $\mathrm{S} 2$. Initial results of orbit-following simulations using experimental data by ASCOT code showed that the signal synchronized with short NB injection was observed in S1 and S2 [18]. A detailed comparison with ASCOT code simulations is presently ongoing.

\section{Summary}

The installation of a Faraday-cup based FILD discussed in this paper, whose aim was the measurement of beam ion losses in W7-X, was a collaborative project between NIFS and IPP. The FILD was installed on the MPM, based on the orbit-following calculation results using LORBIT and ASCOT codes. The location and the size of the aperture and the Faraday-cup were decided using the grid calculation program developed at PPPL. Consequently, a Faraday film was designed with two energy and four pitch angle ranges. Initial measurements of beam ion losses were performed using short-pulse NB blip injections for which barely co-going transit beam ion losses correlated with the NB injections were observed, as was expected from the orbit-following simulations. Moreover, the radial profile of beam ion loss flux showed a peak at approximately $6.127 \mathrm{~m}$. At present, a comparison on the orbit-following simulation is ongoing and is continuing toward an understanding of the beam ion behavior in W7-X.

\section{Acknowledgments}

This work was supported by the NINS program of Promoting Research by Networking among Institutions (Grant Number 01411702), NIFS Stellarator-Heliotron Association Committee (URSX209), and International collaborations with overseas laboratories (UFEX105). This work was carried out within the framework of the EUROfusion Consortium and has received funding from the Euratom research and training programme 2014-2018 and 2019-2020 under grant agreement No 633053. The views and opinions expressed herein do not necessarily reflect those of the European Commission.

\section{References}

[1] A. Fasoli et al., ITER Physics Basis Chapter 5: Physics of energetic ions 2007 Nucl. Fusion 47 S264.

[2] D. S. Darrow et al., Enhancements to the Compact Helical System fast ion loss probe 1999 Rev. Sci. Instrum. 70838.

[3] M. Isobe et al., Design and initial operation of lost fast-ion probe based on thin Faraday films in CHS 2006 Rev. Sci. Instrum. 77 10F508.

[4] K. Ogawa et al., Design of lost fast-ion probe based on thin Faraday films in Heliotron J 2013 Plasma Fusion Res. 82402128. 
[5] S. Yamamoto et al., Faraday-cup-type lost fast ion detector on Heliotron J 2016 Rev. Sci. Instrum. $8711 \mathrm{D} 818$.

[6] D. S. Darrow et al., Fast ion loss diagnostic plans for the National Spherical Torus Experiment 2001 Rev. Sci. Instrum. 72784.

[7] F. E. Cecil et al., The anomalous currents in the front foils of the JET lost alpha diagnostic KA-2 2004 Rev. Sci. Instrum. 7510.

[8] D. S. Darrow et al., Initial results from the lost alpha diagnostics on Joint European Torus 2006 Rev. Sci. Instrum. 77 10E701.

[9] W. P. West et al., Observations of fast ion loss to the plasma facing wall during quiescent H-modes on DIII-D 2005 J. Nucl. Mater. 337-339 420.

[10] S. J. Zweben et al., Loss of alpha-like MeV fusion products from TFTR 1990 Nucl. Fusion 301551.

[11] J. M. Faustin et al., Fast particle loss channels in Wendelstein 7-X 2016 Nucl. Fusion 56092006.

[12] A. Werner et al., Advanced Diagnostics for magnetic and inertial fusion, edited by P. E. Stott et al. Kluwer Academic/Plenum, New York, 2002, p. 137.

[13] R. C. Wolf et al., Major results from the first plasma campaign of the Wendelstein 7-X stellarator 2017 Nucl. Fusion 57102020.

[14] N. Rust, W7-X neutral-beam-injection: selection of the NBI source positions for experiment start-up 2011 Fusion Eng. Des. 86728.

[15] D. Nicolai et al., A multi-purpose manipulator system for W7-X as user facility for plasma edge investigation 2017 Fusion Eng. Des. 123960.

[16] M. Isobe et al., Lorentz Alpha Orbit Calculation in Search of Position Suitable for Escaping Alpha Particle Diagnostics in ITER 2009 J. Plasma and Fusion Res. 8330.

[17] E. Hirvijoki et al., ASCOT: Solving the kinetic equation of minority particle species in tokamak plasmas 2014 Comput. Phys. Commun. 1851310.

[18] S. Äkäslompolo et al., "Validating the ASCOT modeling of NBI fast ions in Wendelstein 7-X," $3^{\text {rd }}$ European Conference on Plasma diagnostics May 6-9 2019, Lisbon, Portugal and to be submitted to Journal of Instrumentations. 